\title{
Metilmercurio en el cabello de población infantil
}

\author{
Llorente Ballesteros Mª. ${ }^{1}$, Vírseda Chamorro I. ${ }^{1}$, Peral Jiménez R. ${ }^{2}$, Sanz Grimaldi M ${ }^{\mathrm{a}} .^{3}$, Ruiz Tejada MaA. ${ }^{4}$, \\ López Colón JL. 5
}

Sanid. mil. 2011; 67 (3): 299-303; ISSN: 1887-8571

\begin{abstract}
RESUMEN
Introducción: El metilmercurio( $\mathrm{MeHg})$ es el compuesto orgánico del mercurio más abundante en el medio ambiente. Éste, es un potente neurotóxico, capaz de atravesar la barrera hematoencefálica y placentaria. Incluso a niveles bajos, puede originar alteraciones en el SNC que afectan a los procesos del desarrollo feto- infantil. La principal fuente de exposición a MeHg es la ingestión de pescado contaminado. Objetivos: La US EPA (Agencia de protección del medio ambiente USA) recomendaba unos niveles de $\mathrm{MeHg}$ en pelo de $1 \mu \mathrm{g} / \mathrm{g}$, mientras que la JEFSA (FAO/OMS) establecía unos niveles de seguridad de $\mathrm{MeHg}$ en pelo de 2,2 $\mu \mathrm{g} / \mathrm{g}$. Dado que España es un gran consumidor de pescado y la vulnerabilidad fetal e infantil al MeHg, se proyectó este estudio de $\mathrm{MeHg}$ en cabello de población infantil de la Comunidad de Madrid. Material y Métodos: Participaron 472 niños, categorizados por edades de: 0-5, 6-10, 11-15 y mayor de 15 y por frecuencia de consumo de pescado semanal (clasificación basada en un cuestionario individualizado de cuatro opciones: no consumidores, consumen 1-2 veces/ semana, consumen 3-4 v/s, consumen más de 4 v/s). Los análisis se hicieron por Espectroscopía de Absorción Atómica y vapor frio. Resultados: Del estudio estadístico con SPSS, se obtuvieron diferencias significativas, en los niveles de MeHg en pelo, entre los que consumen mas de $4 \mathrm{v} / \mathrm{s}$ y los que no consumen $(0,68 \mathrm{vs} 2,34 \mu \mathrm{g} / \mathrm{g})$. Las medianas obtenidas para las distintas categorías de edad fueron: 0,53 $\mu \mathrm{g} / \mathrm{g}, 1,01 \mu \mathrm{g} / \mathrm{g}, 1,56 \mu \mathrm{g} / \mathrm{g}$ y $0,93 \mu \mathrm{g} / \mathrm{g}$. Discusión: En estudios similares se establece que con bajo o ningún consumo de pescado los valores de mercurio en pelo son inferiores a $0,5 \mu \mathrm{g} / \mathrm{g}$. Por el contrario, en España, por tener una dieta rica en consumo de pescado, los niveles de mercurio son superiores con una concentración mínima superior a esa cifra. Conclusión: El estudio concluye que, al menos un 2,5\% de la población infantil de la CAM, excede los límites de seguridad recomendados por la JEFSA (FAO/OMS) y aproximadamente un $30 \%$ supera los límites de seguridad para el MeHg en pelo establecidos por la US EPA.
\end{abstract}

PALABRAS CLAVE: Mercurio, Metilmercurio, Cabello, Niños, Pescado.

\section{Methylmercury in the hair of a children population \\ SUMMARY:}

Introduction: The methylmercury ( $\mathrm{MeHg}$ ) is the most abundant organic compound of mercury in the environment. This is a strong neurotoxic which is able to cross the blood-brain and the placental barriers. Even at low levels, it can originate alterations in the brain that affect the processes of the fetus - children development. The main source of MeHg is the intake of contaminated fish. Objectives: The EPA (US Environmental Protection Agency) recommended MeHg levels in hair of $1 \mu \mathrm{g} / \mathrm{g}$, while the JECFA (FAO / OMS) established safety levels of $\mathrm{MeHg}$ in hair of 2,2 $\mu \mathrm{g} / \mathrm{g}$. This study of $\mathrm{MeHg}$ in hair was carried out in the Region of Madrid's young population because Spain is a great fish consumer, and due to the fetal and children vulnerability to MeHg. Material and methods: 472 children participated, They were categorized by age and by frequency of weekly fish consumption. The age categories were the following: 0-5, 6-10, 11-15 and older than 15, and the frequency of weekly fish consumption was based on an individualized questionnaire with four options (non consumers, consume 1-2 times / week, consume 3-4t/w, consume more than $4 \mathrm{t} / \mathrm{w}$ ). The analyses were made by Cold Vapor Atomic Absorption Espectroscopy. Results: The study carried out with SPSS shows significant differences in the levels of MeHg in hair, among those that consume more than $4 \mathrm{t} / \mathrm{w}$, and those that do not consume $(0,68 \mathrm{vs} 2,34 \mu \mathrm{g} / \mathrm{g})$. The median obtained for the different age categories were: $0,53 \mu \mathrm{g} / \mathrm{g}, 1,01 \mu \mathrm{g} / \mathrm{g}$, $1,56 \mu \mathrm{g} / \mathrm{g}$ and $0,93 \mu \mathrm{g} / \mathrm{g}$. Discussion: Similar studies in countries with low or no consume or fish show that hair mercury levels are below $0.5 \mu \mathrm{g} / \mathrm{g}$. In contrast, in Spain, because its diet rich in fish, the lowest hair mercury levels are higher than that figure. Conclussion: The study concludes that, at least $2,5 \%$ of the children population of the Region of Madrid exceed the limits of safety recommended by JECFA (FAO / OMS) and approximately $30 \%$ exceed the limits of safety for MeHg in hair establish by US EPA.

KEY WORDS: Mercury, Methylmercury, Hair, Children, Fish.

${ }^{1}$ Cte. Farmacéutico.

${ }^{2}$ Cte Cuerpo General Ejército de Tierra.

${ }^{3}$ Diplomado Universitario en Enfermería.

${ }^{4}$ Técnico Especialista en Laboratorio.

${ }^{5}$ TCol Farmacéutico.

Instituto de Toxicología de la Defensa. Madrid. España.

Dirección para correspondencia: María Teresa Llorente Ballesteros. Instituto de Toxicología. Edificio Cuidados Mínimos del Hospital Central de la Defensa Gómez Ulla. Glorieta del Ejército s/n.28047 Madrid. mllobal@oc.mde.es

Recibido: 28 de junio de 2010

Aceptado: 17 de enero de 2011

\section{INTRODUCCIÓN}

La ATSDR 1997 (Agencia para sustancias tóxicas y registro de enfermedades) considera al mercurio como el tercer elemento en el ranking de sustancias peligrosas inmediatamente detrás del plomo y el arsénico ${ }^{1,2}$.

El mercurio ( $\mathrm{Hg})$, metal pesado, está presente en el medio ambiente en diferentes formas: mercurio elemental o metálico $\left(\mathrm{Hg}^{\mathrm{O}}\right)$, mercurio inorgánico $\mathrm{Hg}$ (I) y Hg (II) y mercurio orgánico, formando los compuestos (metil, etil y fenilmercurio). Las diferentes formas 
del mercurio están sujetas a interconversiones principalmente a través de procesos de oxidación-reducción y metilación-desmetilación, procesos que determinan el llamado «ciclo del mercurio» ${ }^{3}$.

El compuesto orgánico del mercurio más abundante en el medio ambiente, es el metilmercurio ( $\mathrm{MeHg}$ ), el cual es generado por los microorganismos (bacterias metanogénicas) mediante el proceso de metilación ${ }^{4}$.

El metilmercurio ( $\mathrm{MeHg}$ ) es tristemente conocido por su neurotoxicidad, debido a los envenenamientos a los que dio lugar en Minamata (Japón) en los años 50, cuando se vertieron en su bahía subproductos orgánicos de $\mathrm{MeHg}$, y en Iraq en 1970, cuando fueron utilizados pesticidas a base de mercurio que causaron graves alteraciones neurológicas, particularmente en niños expuestos en el útero materno, dado que es capaz de atravesar las barreras placentarias y hematoencefálicas; originando parálisis cerebral, ceguera, sordera, retraso mental...5

Más recientemente, diferentes estudios epidemiológicos en las islas Feroe y Nueva Zelanda han confirmado que la exposición, incluso a niveles bajos de metilmercurio, origina también alteraciones en el sistema nervioso que afectan a los procesos del desarrollo feto/ infantil (memoria, atención, aprendizaje... $)^{6-8}$.

Numerosos estudios adicionales de exposición dietética en humanos realizados en diferentes comunidades: Canadá, Madeira (Portugal), Amazonia (Brasil), Nuevo Méjico, Perú, Japón, Camboya, Suecia..., han evidenciado efectos adversos en el desarrollo neurológico de los niños a bajas dosis de $\mathrm{MeHg}^{9-14}$.

La principal fuente de exposición a metilmercurio es la ingestión de pescado contaminado, tanto de agua dulce como de salada y algunos mamíferos marinos. $\mathrm{El} \mathrm{MeHg}$ tiene una rápida difusión y se une a las proteínas de las algas, plancton y otros microorganismos inferiores, éste, se biomagnifica y bioacumula en la cadena alimentaria acuática. Lo anterior motiva que sean los peces que se encuentran en el nivel trófico superior, los llamados predadores, los que posean niveles mayores de mercurio (tiburón, pez espada, caballa, atún grande, lucio, etc., $)^{15}$.

\section{Comités internacionales. Problema de Salud Pública}

Basándose en los informes preliminares de los estudios epidemiológicos, llevados a cabo en las islas Feroe, la organización FAO (Organización para la Agricultura y la Alimentación) y la OMS (Organización Mundial de la Salud) conjuntamente en el Comité Mixto de Expertos en Aditivos Alimentarios (JECFA) redujeron el valor del PTWI (Ingesta Tolerable Semanal Provisional) de MeHg, de 3,3 $\mu \mathrm{g} / \mathrm{kg}$ peso/semana (JECFA 1978) a 1,6 $\mu \mathrm{g} / \mathrm{kg}$ peso/semana (JECFA 2003). Estos valores se corresponden con unos niveles de mercurio en pelo de $2,2 \mu \mathrm{g} / \mathrm{g}^{16-17}$.

Así mismo, la US-EPA (Agencia de Protección del Medio Ambiente de los EEUU), estableció la Dosis de Referencia de Ingestión de $\mathrm{MeHg}$ (RfD) en 0,1 $\mu \mathrm{g} / \mathrm{kg}$ peso/día (US-EPA 1997). Estos valores se corresponden con unos niveles de mercurio en pelo de $1 \mu \mathrm{g} / \mathrm{g}^{18-20}$.

La Comisión Europea para la Seguridad Alimentaria (EFSA) recomendaba en 2004, tener en cuenta el consumo de mercurio a través del pescado en los grupos vulnerables (mujeres embarazadas, madres lactantes y niños) pues su ingesta, gracias al MeHg que contienen, podría aumentar el riesgo de lesión en el SNC (Sistema Nervioso Central) fetal e infantil. En 2008 este mismo organismo reco- mendó a las mujeres en edad fértil, embarazadas, madres lactantes y niños,» comer menos de $100 \mathrm{~g}$ por semana de pescados predadores». Esta institución, insta a cada país a regular en su legislación el consumo de pescado en los grupos vulnerables ${ }^{21-22}$.

La Agencia Española de Seguridad Alimentaria y Nutrición $(\mathrm{AESAN})^{23}$ hizo suya la guía de recomendaciones establecidas por la OMS, junto con la UNEP (Programa de Medio Ambiente de las Naciones Unidas) en 2008, que informa sobre el potencial impacto de la exposición a mercurio en las poblaciones más vulnerables.

\section{OBJETIVOS}

Basándose en estimaciones nutricionales obtenidas a partir de encuestas de población (NHANES- Nacional Health and Nutrition Examination Survey -NCSH 03), se cree que, en la actualidad, aproximadamente el $7 \%$ de las mujeres fértiles de EEUU consume cantidades de $\mathrm{MeHg}$ superiores a las consideradas seguras por la US EPA 1997 y NRC 200024-27.

España cuenta con un elevado consumo de pescado, aproximadamente $70 \mathrm{~g}$ /persona (Martin 2002) ${ }^{28}$, situada, esta cifra, muy por encima del promedio de la Unión Europea (10 g/persona en los Países Bajos a 80 g/persona en Noruega.)(EFSA 2004). Por ello y teniendo en cuenta que:

I.- Estos parámetros hacen referencia a una población adulta.

II. - El mayor consumo por $\mathrm{Kg}$ de peso se da en los niños ${ }^{29}$.

III.- Es el pescado un elemento destacado en la dieta Mediterránea $^{30-32} \mathrm{y}$

IV.- La mayor vulnerabilidad fetal e infantil a la exposición al $\mathrm{MeHg}$.

Se puede predecir que muchos de nuestros niños ingieren una cantidad de $\mathrm{MeHg}$ que supera los límites de Seguridad recomendados por la US EPA y por la JECFA (FAO/OMS) $)^{33-35}$.

Sobre lo anteriormente expuesto, y dado que el $\mathrm{Hg}$ en pelo, es el biomarcador preferido para evaluar la exposición a $\mathrm{Hg}$ en periodos largos de tiempo. Se proyectó este estudio para investigar el contenido de MeHg en el pelo de población infantil de la Comunidad de Madrid.

\section{MATERIAL Y MÉTODOS}

\section{Población estudio}

Se realizó un estudio descriptivo transversal.

La población de la Comunidad de Madrid según el padrón municipal de 2009 es de 6.386.000. En ésta, la población infantil/juvenil menor de 18 años es aproximadamente de 1.255.000.

La selección de la muestra se llevó a cabo de forma consecutiva no probabilística a partir de tres colegios elegidos al azar en la Comunidad de Madrid.

Para el estudio, se elaboró un cuestionario de frecuencia de consumo de pescado semanal, en el que se solicitaban además otras variables: edad, sexo, peso, talla y posibles patologías en el periodo comprendido entre abril de 2008 a diciembre de 2009.

Se contactó directamente con cada uno de los niños y para su inclusión en el estudio los padres y/o responsables dieron su auto- 
Tabla 1. Mercurio total en pelo ( $\mu \mathrm{g} / \mathrm{g})$ en población infantil.

\begin{tabular}{lccccc}
\hline & Núm. casos & Media & Percentil 25 & Percentil 50 & Percentil 75 \\
\hline No consumen pescado & 39 & 0,88 & 0,26 & 0,68 & 1 \\
1-2 veces semana & 225 & 1,17 & 0,43 & 0,85 & 1,38 \\
3-4 veces semana & 172 & 1,42 & 0,63 & 1,09 & 1,75 \\
Mayor de 4 veces semana & 22 & 3,67 & 1,32 & 2,34 & 3,5 \\
\hline
\end{tabular}

Tabla 2. Diferencia de $H g(\mu g / g)$ en pelo en distintos grupos de edad en relación al consumo de pescado.

\begin{tabular}{lccccccccc}
\hline \multirow{2}{*}{ Consumo } & \multicolumn{2}{c}{$\mathbf{1}$ a 5 años } & \multicolumn{2}{c}{$\mathbf{6}$ a 10 años } & \multicolumn{2}{c}{$\mathbf{1 1}$ a 15 años } & \multicolumn{2}{c}{ Mayores de 15 } \\
& $\mathrm{Hg}$ & $\mathrm{MeHg}$ & $\mathrm{Hg}$ & $\mathrm{MeHg}$ & $\mathrm{Hg}$ & $\mathrm{MeHg}$ & $\mathrm{Hg}$ & $\mathrm{MeHg}$ \\
\cline { 2 - 9 } Superior a 4 veces por semana & 1,87 & 1,5 & 3,3 & 2,64 & 1,98 & 1,58 & 2,75 \\
No Consumen & 0,5 & 0,4 & 0,7 & 0,56 & 0,68 & 0,54 & 0,59 & 0,47 \\
\hline
\end{tabular}

* El MeHg es estimado como el 80\% del Hg

rización mediante consentimiento informado. El número de participantes fue de 472, agrupados en cuatro categorías: de 0 a 5 , de 6 a 10 , de 11 a 15 y mayores de 15 años.

\section{Recolección de muestras (cabello)}

Para el ensayo fue requerido un mínimo de 5-10 mg de pelo. La recolección se realizó cortando de la región occipital, lo más cercano a la raíz, un mechón de cabello del diámetro aproximado de un lápiz ${ }^{36,37}$. Cada muestra se introdujo en un envase aséptico de plástico. Posteriormente se envió al laboratorio para su análisis.

\section{Análisis de muestras}

Las muestras fueron pretratadas con una solución de acetona y lavado posterior con agua MiliQ para evitar posibles contaminaciones. Seguidamente fueron sometidas a un proceso de digestión ácida con un mezcla de sulfúrico y nítrico $(30: 70)$ y digeridas en estufa a $\left(60^{\circ}+/-5^{\circ}\right)$ por un periodo de 16 horas.

La metodología empleada fue espectroscopía de absorción atómico y vapor frío con un equipo de la casa comercial Perkin Elmer mod. FIMS 400.

Las muestras fueron medidas por triplicado y aceptadas cuando la repetibilidad de las medidas era menor al $10 \%$.

\section{Estadística}

Para el análisis estadístico de los datos se utilizó el programa estadístico SPSS (V-15). Para el tratamiento de los datos, la variable frecuencia de consumo de pescado se dividió en las siguientes categorías: no consumidores, consumidores de una o dos veces por semana, consumidores de tres o cuatro veces por semana y consumidores de más de cuatro veces.

El análisis descriptivo de la muestra nos hizo ver que se trataba de una distribución no normal. Como estadísticos descriptivos se seleccionaron, por tanto, la mediana y los percentiles.

Se utilizaron pruebas no paramétricas: U de Mann-Whitney y Kruskal-Wallis para el análisis de los datos.

\section{RESULTADOS}

De los 472 participantes, un 97\% fueron válidos.

En la tabla 1, se muestras los percentiles 25, 50 y 75, así como las medias aritméticas que fueron incluidas para poderse comparar con otros resultados de la bibliografía consultada ${ }^{9,25,38}$.

Se observa la existencia de diferencias significativas $(\mathrm{p}<0,05)$, entre el mercurio que existe en los no consumidores de pescado y aquellos que consumen más de cuatro veces por semana $(0,68 \mu \mathrm{g} / \mathrm{g}$ vs $2,34 \mu \mathrm{g} / \mathrm{g})$. Así mismo, ésto se verifica en los distintos grupos de edad.

\section{DISCUSIÓN}

Se ha elegido la muestra de cabello para determinar el $\mathrm{Hg}$, dado la facilidad de su obtención, y porque es considerado el biomarcador más adecuado para evaluar la exposición al $\mathrm{Hg}$ en prolongados periodos de tiempo (NRC-2000).

Considerando los estudios similares llevados a cabo en otros países, se ha establecido que el $\mathrm{Hg}$ en poblaciones que no consumen pescado o bien lo consumen con muy escasa frecuencia presentan valores de $\mathrm{Hg}$ en pelo inferiores a $0,5 \mu \mathrm{g} / \mathrm{g}$.

Por el contrario, países como España con una dieta rica en pescado manifiestan unos valores de $\mathrm{Hg}$ en pelo superiores, como se significa en este estudio ${ }^{39,40}$ :

- Niños de 1 a 5 años, mediana: $0,53 \mu \mathrm{g} / \mathrm{g}$

- Niños de 6 a 10 años, mediana: $1,01 \mu \mathrm{g} / \mathrm{g}$

- Niños de 11 a 15 años, mediana: $1,56 \mu \mathrm{g} / \mathrm{g}$

- Niños mayores de 15 años, mediana: $0,93 \mu \mathrm{g} / \mathrm{g}$

Una limitación al estudio puede encontrarse en el menor número de niños presentes en los grupos que no consumen pescado y en aquellos que consumen pescado más de 4 veces semana, no alcanzándose en estos casos el tamaño muestral idóneo.

\section{CONCLUSIÓN}

Se ha podido verificar que al menos un 2,5\% (31.000) de la población infantil de la Comunidad de Madrid, excede los límites de 


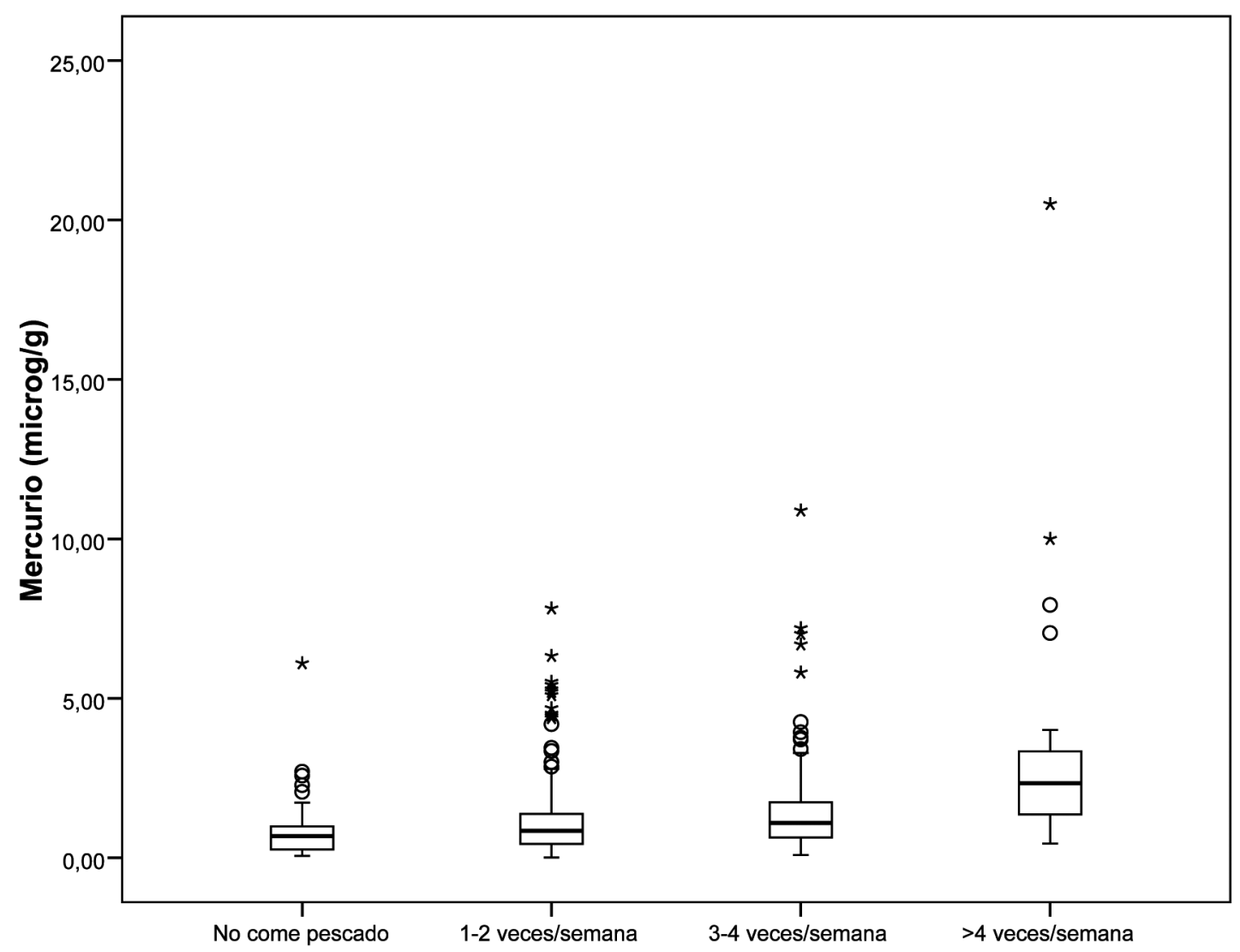

Figura 1. Box plot representando la concentración de Hg en pelo en relación al consumo de pescado.

seguridad recomendados por la JECFA (FAO/OMS) en $2,2 \mu \mathrm{g} / \mathrm{g}$ y un porcentaje muy superior a éste, llegando a alcanzar el 30\% (376.500 menores de 18 años) supera los límites de seguridad para el $\mathrm{MeHg}$, establecidos por la US EPA en $1 \mu \mathrm{g} / \mathrm{g}$.

\section{BIBLIOGRAFÍA}

1. ATSDR (U.S.Department of Health and Human Services. Agency for Toxic Substances and Disease Registry). Toxicological profile for mercury: A National review of exposure events: 2009.

2. ATSDR (U.S.Department of Health and Human Services. Agency for Toxic Substances and Disease Registry). Toxicological profile for mercury: 1999.

3. Clarkson TW. The toxicology of mercury. Crit. Rev. Clin Lab Sci 1997;34:369403

4. Gónzalez F, Schalscha E, Becerra .J, Silva M. Mercury in a marine trophic chain Bull Environ Contam Toxicol. 2002;68:448-454

5. Trasande L, Landrigan P, Schechter C. Public Health and economics consecuences of methylmercury toxicity to the developing brain. Environt. Healt Persp 2005; 113: 5 .

6. Grandjean P. late insights into early origins of disease. Basic Clin Pharmacol Toxicol 2008;102:94-9.

7. Grandjean P., Wheihe P, White RF, debes F, Araki S, Yokoyama K, et al. Cognitive defeicit in 7- year - old children whit prenatal exposure to methylmercury. Neurotoxicol Teratol 1997; 19: 417-28.

8. Holmes P, James K.A.F, Levy L. S. Is low- level environmental mercury exposure of concern to human health. Scienc. of the Total Environment.

9. Becker k, Kaus S, Krause C, Leopon P, Schulz C, Seiwert M, et al 2002. German Environmental survey 1998(Ger ES III): environmemtal pollutants in blood of German polutation . Int J Hyg Environ health 205:297-3008.
10. Bjornberg K A, Vahter M, Grawe k.P, Berglund M. 2005. Methyl mercury exposure in Swedish women with high fish consumption. Sci Total environ 341:45-52.

11. Innis SM, Palaty J, Vaghri Z, Lockith G. 2006. Increased levels of mercury associated with high intakes among children from Vancouver. Canada J. Pedriatr. 148:759-763.

12. Murata K, Sakamoto M, Nakai K, Weihe P, Dakeishi, Iwata T, Liu X, Ohmo T, Kurosawa T, Kamiya K, Satoh H. Effects of methylmercury on neurodevelopment in Japanese children in relation to the Madeira Study. Int. Arch. Occup.Environ Health 2004; 77: 571-579.

13. Tetsuro a, Kunito T, Iwata H, Monirith I, Seang Tana T, Subramanian A, Tanabe S. Mercury contamination in human Hair and fish from Cambodia: levels, specific accumulation and risk assesment. Environmental Pollution 2005; 134: 79-86.

14. Tetsuya E, Koichi H. Hair Mercury Levels in hair samples from residents of Taiji, a Japanese whaling town. Marine pollution Bulletin 2009; in press.

15. Hightower J, Moore D, Mercury levels in high-End consumers of fish. Environt. Healt Persp 2003; 111: 2.

16. JECFA (Joint Food and Agriculture Organization of the United nations/world Health Organization (FAO/OMS). Expert Committee on Food Additives).Sixty seven meeting in Rome 20-29 June 2006.

17. WHO (World Health Organisation International Programme on Chemical Safety). Methylmercury (addendum); 2004.

18. US EPA (US Environment Protection agency)Mercury Study Report to Congress; 1997.

19. WHO (World Health Organisation). Exposure a Mercury: a major public health concern;2007.

20. Yasutake A, Matsumoto m, Yamaguchi N, Hachiya N. Current hair mercury levels in Japanese for estimation of methylmercury exposure. J. health Sci 2004; 50: 120-125.

21. European Commission Directorate General Environment. Mercury. European Commission DG Env; 2009.

22. European Commission Directorate General Environment. Methyl mercury in fish and fishery products; 2008 . 


\section{Metilmercurio en el cabello de población infantil}

23. Agencia española de Seguridad Alimentaria (AESA). Nota informativa de la AESA (17-06-04) sobre mercurio y metil mercurio en productos pesqueros. Ministerio de Sanidad y Consumo España .AESA, Madrid 17 junio 2004.

24. Mahaffey K, Clickner R, Bodurow C. Blood organic mercury and dietary mercury intake: National health and nutrition examination survey, 1999 and 2000. Environmental Health Perpestive 2004: 112; 5.

25. Mcdowell M, Dillon C, Osterloh J, Bolger P, Pellizari E, Fernando R, monytes de Oca R, Schober S, Sinks T, Jones R, Mahaffey K. Hair Mercury Levels in US Children and women of childbearing age: Reference reange data from NHAMES 1999-2000. Environt. Healt Persp 2004; 112: 11.

26. National Research Council. Toxicological effects of methylmercury . A report of the Committee on the toxicological Effects of Methylmercury, Board on Environmental studies and Toxicology. Washington, DC: National Academy Press 2000.

27. NCHS.2003. national health and Nutrition examination survey. Hyattsville, Md National Center for Health Statistic.

28. Martin VJ. 2002. Consumo de productos de la pesca. Distrución territorial. Distribución y consume. 65:73-79.

29. Sahuquillo I, Lagarda MJ, Silvestre MD .Methyl mercury determination in fish and seafood products and estimated daily intake for the Spanish population. Food Addit Contam 2007;24:869-876.

30. Ortega J.A, Ferris J, Cánovas A, garcía J. Neurotóxicos medioambientales (y II). Metales: efectos adversos en el sistema nervioso fetal y posnatal. Acta Pediatric Esp. 2005; 63: 182-192.

31. Rubio C, Gutierrez A, Burgos A, Hardisson. Total dietary intake of mercury in the canary Islands, Spain Food Additives and Contaminants2008; 25: 946-952.

32. UrietaI, Jalón M, Eguilero I. Food surveillance in Basque country (Spain). Food Addit Contam. 1996;13:289-352.
33. Martí-Cid R, Llobet J.M, Castell, Domingo JL. Dietary intake of arsenic, cadmium, Mercury and lead by the population of Catalonia, Spain. Biol Trace Elem Res (2008) 125:120-132.

34. Ortega-Garcia JA, RodrigueZ K, Calatayud M, Martin M, Velez D, Devesa V, Sanchez- Alarcón C, Torres A, Galindo C, Gil- Vazquez JM, Sánchez M, Sanchez Solis M, Alfonso B, Romero F. Estimated intake levels of methyl mercury in children, childbearing age and pregnant women in a Mediterranean region, Murcia, Spain . Eur J. Pediatr 2009; 168:1075-1080.

35. Sanzo JM, Dorronsoro M, Amiano P, Aguinagalde F, Azpiri P, Estimation y validation of mercury intake associated with fish comsumption in an EPIC cohort of Spain. Public Health Nutr. 2001; 4: 981-988.

36. Srogi K. Mercury content of Hair in differents populations relative to fish consumption. Rev Environ Contam Toxicolo 2007: 189: 107-130.

37. Cernichiari E, Brewer R, Myers GJ, Marsh DO, Laphan LW, Cox C. Monitoring methylmercury during pregnancy: maternal hair predicts fetal brain exposure. Neurotoxicology 1995;16: 705-710.

38. Grandjean P. Methylmercury toxicity and functional programming. Reprod Toxicol 2007;23:414-20.

39. Moreiras O, Cuadrado C, Kumpulainem JT, Carbajal A, Ruiz-Roso B. Intake of contaminants, heavy metals and nutrients with potential toxicity via total diet in four geographical areas of Spain. FAO regional Office for Europe, REU Technical series 49. Trace elements, natural antioxidants and contaminants in European foods and diets . Rome: FAO, 1996; 59-92.

40. Schumacher M, Batiste J, Bosque MA, Domingo JL, Corbella J. Mercury concentrations in marine species from the coastal area of Tarragona province, Spain Dietary intake of mercury through fish and seafood consumption. Sci Total Environ. 1994;156: 269-273 